\title{
Conjugate boundary condition, hidden particles, and gauge-Higgs inflation
}

\author{
Yugo Abed1, Yuhei Goto"2, Yoshiharu Kawamura 43 , \\ and Yasunari Nishikawa包 \\ ${ }^{a}$ Graduate School of Science and Engineering, Shimane University, \\ Matsue 690-8504, Japan \\ a Department of Physics, Tokyo Institute of Technology, Tokyo \\ 152-8551, Japan \\ ${ }^{b}$ Department of Physics, Shinshu University, Matsumoto 390-8621, \\ Japan
}

\begin{abstract}
We propose an idea that hidden particles can be separated according to gauge quantum numbers from the visible ones by the difference of boundary conditions on extra dimensions. We formulate 5-dimensional gauge theories yielding conjugate boundary conditions besides ordinary ones on $S^{1} / Z_{2}$, and examine physical implications concerning hidden particles on an extension of the standard model coexisting different types of boundary conditions. A model with conjugate boundary conditions is applied on a gauge-Higgs inflation scenario.
\end{abstract}

\footnotetext{
${ }^{1}$ E-mail: yugo@riko.shimane-u.ac.jp

${ }^{2}$ E-mail: 14st302a@shinshu-u.ac.jp

${ }^{3}$ E-mail: haru@azusa.shinshu-u.ac.jp

${ }^{4}$ E-mail: 15sm210a@shinshu-u.ac.jp
} 


\section{Introduction}

There are several riddles that cannot be solved in the framework of the standard model (SM), but any evidences from new physics such as supersymmetry, compositeness and extra dimensions have not been discovered. Here, the SM means the extended model including the modifications with massive neutrinos.

One of big issues in physics beyond the SM is to disclose the identity of unknown particles such as dark matter and inflaton. Because it is hard to detect such hidden particles directly, they are supposed to interact with the SM particles weakly. A typical example is a scenario based on the Weakly Interacting Massive Particle (WIMP) assumption for dark matter, and possible dark matter candidates are the lightest superparticle (LSP) in a supersymmetric extension of the SM and the lightest Kaluza-Klein mode in an extra-dimensional one.

In this paper, we consider an extreme case that hidden particles are singlets of the SM gauge symmetries and the SM particles are singlets of gauge symmetries in a hidden sector. Here, the hidden sector stands for a sector that contains singlets of the SM gauge group besides graviton. In this case, we have a question "how is such a subtle separation of gauge quantum numbers realized naturally?" We present an idea that hidden particles can be separated according to gauge quantum numbers from the visible ones by the difference of boundary conditions (BCs) on extra dimensions, as a possible answer. To illustrate our idea, we construct 5-dimensional (5D) gauge theories yielding conjugate BCs besides ordinary ones on $S^{1} / Z_{2}$, and show that the separation of visible and hidden particles can be realized in gauge interactions using a $5 \mathrm{D}$ extension of the SM with an extra $U(1)$ gauge symmetry coexisting different types of BCs. Furthermore, we study models that hidden particles relating to conjugate $\mathrm{BCs}$ are identified with dark matter or inflaton.

This paper is organized as follows. In the next section, we formulate a 5D $U(1)$ gauge theory yielding conjugate $\mathrm{BCs}$ on $S^{1} / Z_{2}$. We examine physical implications on a theory coexisting different types of BCs in Sect. 3, and apply a model with conjugate BCs on a gauge-Higgs inflation scenario in Sect. 4. In the last section, we give conclusions and discussions.

\section{Conjugate boundary condition on $S^{1} / Z_{2}$}

The space-time is assumed to be factorized into a product of 4-dimensional (4D) Minkowski space-time $M^{4}$ and the orbifold $S^{1} / Z_{2}$, whose coordinates are denoted by $x^{\mu}$ (or $\left.x\right)(\mu=0,1,2,3)$ and $y\left(=x^{5}\right)$, respectively. The $5 \mathrm{D}$ notation $x^{M}(M=$ $0,1,2,3,5)$ is also used. The $S^{1} / Z_{2}$ is obtained by dividing the circle $S^{1}$ (with the identification $y \sim y+2 \pi R$ ) by the $Z_{2}$ transformation $y \rightarrow-y$. Then, the point $y$ is identified with $-y$ on $S^{1} / Z_{2}$, and the space is regarded as an interval with length $\pi R$ ( $R$ being the radius of $S^{1}$ ).

In the following, we formulate a $5 \mathrm{D} U(1)$ gauge theory with ordinary $\mathrm{BCs}$ and that with conjugate $\mathrm{BCs}$, respectively. 


\subsection{Ordinary boundary condition}

For completeness, we explain ordinary BCs using the 5D $U(1)$ gauge theory whose Lagrangian density is given by

$$
\mathscr{L}_{B}=\left(D_{M} \varphi\right)^{*}\left(D^{M} \varphi\right)-m_{\varphi}^{2}|\varphi|^{2}+\bar{\psi}\left(i \Gamma^{M} D_{M}-m_{\psi}\right) \psi-\frac{1}{4} B_{M N} B^{M N},
$$

where $D_{M}=\partial_{M}-i g_{5} q_{\varphi} B_{M}$ for a complex scalar field $\varphi=\varphi(x, y), *$ stands for the complex conjugation, $D_{M}=\partial_{M}-i g_{5} q_{\psi} B_{M}$ for a $5 \mathrm{D}$ spinor field $\psi=\psi(x, y)$, and $m_{\varphi}$ and $m_{\psi}$ are masses of $\varphi$ and $\psi$, respectively. The $\bar{\psi}$ is the Dirac conjugate of $\psi$ defined by $\bar{\psi} \equiv \psi^{\dagger} \gamma^{0}$. The $g_{5}$ is a $5 \mathrm{D}$ gauge coupling constant, $B_{M}\left(=B_{M}(x, y)\right)$ is a $5 \mathrm{D} U(1)$ gauge boson, $B_{M N}$ is the gauge strength defined by $B_{M N}=\partial_{M} B_{N}-\partial_{N} B_{M}$, and $q_{\varphi}$ and $q_{\psi}$ are $U(1)$ charges of $\varphi$ and $\psi$, respectively. The 5D gamma matrices $\Gamma^{M}$ are given by

$$
\Gamma^{\mu}=\gamma^{\mu}, \quad \Gamma^{5}=i \gamma^{5},
$$

using 4D gamma matrices $\gamma^{\mu}$ and $\gamma^{5}=i \gamma^{0} \gamma^{1} \gamma^{2} \gamma^{3}$, and they satisfy the algebraic relation $\left\{\Gamma^{M}, \Gamma^{N}\right\}=2 \eta^{M N}$ with $\eta^{M N}=\operatorname{diag}(1,-1,-1,-1,-1)$.

From the requirement that the Lagrangian density should be invariant under the translation $y \rightarrow y+2 \pi R$ and the $Z_{2}$ transformation $y \rightarrow-y$ or it should be a singlevalued function on the $5 \mathrm{D}$ space-time, the BCs of fields on $S^{1} / Z_{2}$ are determined up to some parameters such as intrinsic $Z_{2}$ parities.

Because the derivative $\partial_{M}$ is invariant under $y \rightarrow y+2 \pi R, D_{M}$ should also be invariant. Under $y \rightarrow-y, \partial_{M}$ transforms as $\partial_{\mu} \rightarrow \partial_{\mu}$ and $\partial_{5} \rightarrow-\partial_{5}$, and hence $D_{M}$ is ordinarily supposed to transform as

$$
D_{\mu} \rightarrow D_{\mu}, \quad D_{5} \rightarrow-D_{5} .
$$

Then, the BCs of $B_{M}$ are determined as

$$
\begin{aligned}
& B_{M}(x, y+2 \pi R)=B_{M}(x, y), \\
& B_{\mu}(x,-y)=B_{\mu}(x, y), \quad B_{5}(x,-y)=-B_{5}(x, y),
\end{aligned}
$$

and $B_{M}$ are given by the Fourier expansions:

$$
\begin{aligned}
& B_{\mu}(x, y)=\frac{1}{\sqrt{2 \pi R}} B_{\mu}^{(0)}(x)+\frac{1}{\sqrt{\pi R}} \sum_{n=1}^{\infty} B_{\mu}^{(n)}(x) \cos \frac{n y}{R}, \\
& B_{5}(x, y)=\frac{1}{\sqrt{\pi R}} \sum_{n=1}^{\infty} B_{5}^{(n)}(x) \sin \frac{n y}{R} .
\end{aligned}
$$

Note that a zero mode (or a $y$-independent part) of $B_{5}$ is absent.

The BCs of $\varphi$ and $\psi$ are determined as

$$
\begin{aligned}
& \varphi(x, y+2 \pi R)=e^{i \beta_{\varphi}} \varphi(x, y), \quad \varphi(x,-y)=\eta_{\varphi} \varphi(x, y), \\
& \psi(x, y+2 \pi R)=e^{i \beta_{\psi}} \psi(x, y), \quad \psi(x,-y)=\eta_{\psi} i \Gamma^{5} \psi(x, y),
\end{aligned}
$$


where $\beta_{\varphi}$ and $\beta_{\psi}$ take 0 or $\pi$. The $\eta_{\varphi}$ and $\eta_{\psi}$ are the intrinsic $Z_{2}$ parity of $\varphi$ and $\psi$, respectively, and they take 1 or -1 . From (2.8), $\varphi$ is given by the Fourier expansions:

$$
\begin{aligned}
& \varphi(x, y)=\frac{1}{\sqrt{2 \pi R}} \varphi^{(0)}(x)+\frac{1}{\sqrt{\pi R}} \sum_{n=1}^{\infty} \varphi^{(n)}(x) \cos \frac{n y}{R}, \\
& \varphi(x, y)=\frac{1}{\sqrt{\pi R}} \sum_{n=1}^{\infty} \varphi^{(n)}(x) \sin \frac{n y}{R} \\
& \varphi(x, y)=\frac{1}{\sqrt{\pi R}} \sum_{n=1}^{\infty} \varphi^{(n)}(x) \cos \frac{\left(n-\frac{1}{2}\right) y}{R}, \\
& \varphi(x, y)=\frac{1}{\sqrt{\pi R}} \sum_{n=1}^{\infty} \varphi^{(n)}(x) \sin \frac{\left(n-\frac{1}{2}\right) y}{R}
\end{aligned}
$$

for $\left(\beta_{\varphi}, \eta_{\varphi}\right)$ is $(0,1),(0,-1),(\pi, 1)$ and $(\pi,-1)$, respectively. In a similar way, from (2.9), $\psi$ is also given as Fourier expansions. Here, we give only a case with $m_{\psi}=0$, $\beta_{\psi}=0$ and $\eta_{\psi}=1$,

$$
\begin{aligned}
& \psi_{\mathrm{L}}(x, y)=\frac{1}{\sqrt{2 \pi R}} \psi_{\mathrm{L}}^{(0)}(x)+\frac{1}{\sqrt{\pi R}} \sum_{n=1}^{\infty} \psi_{\mathrm{L}}^{(n)}(x) \cos \frac{n y}{R}, \\
& \psi_{\mathrm{R}}(x, y)=\frac{1}{\sqrt{\pi R}} \sum_{n=1}^{\infty} \psi_{\mathrm{R}}^{(n)}(x) \sin \frac{n y}{R}
\end{aligned}
$$

where $\psi_{\mathrm{L}}$ and $\psi_{\mathrm{R}}$ are 2-component spinor fields whose $4 \mathrm{D}$ chirality (the eigenvalue of $\gamma^{5}$ ) is -1 and 1 , respectively.

\subsection{Conjugate boundary condition}

Let us study another BCs using the 5D $U(1)$ gauge theory whose Lagrangian density is given by

$$
\mathscr{L}_{C}=\left(D_{M} \tilde{\varphi}\right)^{*}\left(D^{M} \tilde{\varphi}\right)-m_{\tilde{\varphi}}^{2}|\tilde{\varphi}|^{2}+\overline{\tilde{\psi}}\left(i \Gamma^{M} D_{M}-m_{\tilde{\psi}}\right) \tilde{\psi}-\frac{1}{4} C_{M N} C^{M N},
$$

where $D_{M}=\partial_{M}-i \tilde{g}_{5} \tilde{q}_{\tilde{\varphi}} C_{M}$ for a complex scalar field $\tilde{\varphi}=\tilde{\varphi}(x, y), D_{M}=\partial_{M}-i \tilde{g}_{5} \tilde{q}_{\tilde{\psi}} C_{M}$ for a $5 \mathrm{D}$ spinor field $\tilde{\psi}=\tilde{\psi}(x, y)$, and $m_{\tilde{\varphi}}$ and $m_{\tilde{\psi}}$ are masses of $\tilde{\varphi}$ and $\tilde{\psi}$, respectively. The $\tilde{g}_{5}$ is a $5 \mathrm{D}$ gauge coupling constant, $C_{M}$ is a $5 \mathrm{D} U(1)$ gauge boson, $C_{M N}$ is the gauge strength defined by $C_{M N}=\partial_{M} C_{N}-\partial_{N} C_{M}$, and $\tilde{q}_{\tilde{\varphi}}$ and $\tilde{q}_{\tilde{\psi}}$ are $U(1)$ charges of $\tilde{\varphi}$ and $\tilde{\psi}$, respectively.

Under $y \rightarrow y+2 \pi R$, let $D_{M}$ be invariant. Under $y \rightarrow-y$, let $D_{M}$ transform as

$$
D_{\mu} \rightarrow D_{\mu}^{*}, \quad D_{5} \rightarrow-D_{5}^{*}
$$

Then, the BCs of $C_{M}$ are determined as

$$
C_{M}(x, y+2 \pi R)=C_{M}(x, y)
$$




$$
C_{\mu}(x,-y)=-C_{\mu}(x, y), \quad C_{5}(x,-y)=C_{5}(x, y)
$$

and $C_{M}$ are given by the Fourier expansions:

$$
\begin{aligned}
& C_{\mu}(x, y)=\frac{1}{\sqrt{\pi R}} \sum_{n=1}^{\infty} C_{\mu}^{(n)}(x) \sin \frac{n y}{R}, \\
& C_{5}(x, y)=\frac{1}{\sqrt{2 \pi R}} C_{5}^{(0)}(x)+\frac{1}{\sqrt{\pi R}} \sum_{n=1}^{\infty} C_{5}^{(n)}(x) \cos \frac{n y}{R} .
\end{aligned}
$$

Note that $C_{5}$ has even $Z_{2}$ parities, and its zero mode $C_{5}^{(0)}$ survives and becomes a dynamical field.

The BCs of $\tilde{\varphi}$ and $\tilde{\psi}$ are determined as

$$
\begin{aligned}
& \tilde{\varphi}(x, y+2 \pi R)=e^{i \beta_{\tilde{\varphi}}} \tilde{\varphi}(x, y), \quad \tilde{\varphi}(x,-y)=\tilde{\varphi}^{*}(x, y), \\
& \tilde{\psi}(x, y+2 \pi R)=e^{i \beta_{\tilde{\psi}}} \tilde{\psi}(x, y), \quad \tilde{\psi}(x,-y)=i \tilde{\psi}^{c}(x, y),
\end{aligned}
$$

where $\beta_{\tilde{\varphi}}$ and $\beta_{\tilde{\psi}}$ are arbitrary real constants and $\tilde{\psi}^{c}=e^{i \gamma_{c}} \Gamma^{2} \tilde{\psi}^{*}$. The $\tilde{\psi}^{c}$ corresponds to a charge conjugation of $\tilde{\psi}$ on $4 \mathrm{D}$ space-time, and $\gamma_{\mathrm{c}}$ is an arbitrary real number. A set of BCs (2.18), (2.19), (2.22) and (2.23) corresponds to that in a $U(1)$ case of the orbifold breaking by outer automorphisms [1], and we refer to such BCs relating particles with a representation $\mathbf{R}$ to that with the conjugated one $\overline{\mathbf{R}}$ as conjugate $B C s$ [2].

We find that the complex scalar field part of $\mathscr{L}_{C}$ is single-valued from the translation and the $Z_{2}$ transformation properties such that

$$
\begin{aligned}
\left(\partial_{M}-i \tilde{g}_{5} \tilde{q}_{\tilde{\varphi}} C_{M}(x, y)\right) \tilde{\varphi}(x, y) \rightarrow & \left(\partial_{M}-i \tilde{g}_{5} \tilde{q}_{\tilde{\varphi}} C_{M}(x, y+2 \pi R)\right) \tilde{\varphi}(x, y+2 \pi R) \\
& =e^{i \beta_{\tilde{\varphi}}}\left(\partial_{M}-i \tilde{g}_{5} \tilde{q}_{\tilde{\varphi}} C_{M}(x, y)\right) \tilde{\varphi}(x, y)
\end{aligned}
$$

and

$$
\begin{aligned}
\left(\partial_{\mu}-i \tilde{g}_{5} \tilde{q}_{\tilde{\varphi}} C_{\mu}(x, y)\right) \tilde{\varphi}(x, y) \rightarrow & \left(\partial_{\mu}-i \tilde{g}_{5} \tilde{q}_{\tilde{\varphi}} C_{\mu}(x,-y)\right) \tilde{\varphi}(x,-y) \\
& =\left(\partial_{\mu}+i \tilde{g}_{5} \tilde{q}_{\tilde{\varphi}} C_{\mu}(x, y)\right) \tilde{\varphi}^{*}(x, y) \\
& =\left(D_{\mu} \tilde{\varphi}(x, y)\right)^{*} \\
\left(\partial_{5}-i \tilde{g}_{5} \tilde{q}_{\tilde{\varphi}} C_{5}(x, y)\right) \tilde{\varphi}(x, y) \rightarrow & \left(-\partial_{5}-i \tilde{g}_{5} \tilde{q}_{\tilde{\varphi}} C_{5}(x,-y)\right) \tilde{\varphi}(x,-y) \\
& =-\left(\partial_{5}+i \tilde{g}_{5} \tilde{q}_{\tilde{\varphi}} C_{5}(x, y)\right) \tilde{\varphi}^{*}(x, y) \\
& =-\left(D_{5} \tilde{\varphi}(x, y)\right)^{*},
\end{aligned}
$$

respectively. From (2.22), $\tilde{\varphi}$ is given by the Fourier expansion:

$$
\tilde{\varphi}(x, y)=\frac{1}{2 \sqrt{\pi R}} \sum_{n=-\infty}^{\infty} \tilde{\varphi}^{(n)}(x) e^{i \frac{2 \pi n+\tilde{\varphi}_{\tilde{\varphi}}}{2 \pi R}},
$$

where $\tilde{\varphi}^{(n)}(x)$ are 4D real scalar fields $\left(\tilde{\varphi}^{(n) *}(x)=\tilde{\varphi}^{(n)}(x)\right)$. 
In a similar way, we find that the spinor field part of $\mathscr{L}_{C}$ is also single-valued from the transformation properties such that

$$
\begin{aligned}
& \overline{\tilde{\psi}}(x, y)\left\{i \Gamma^{M}\left(\partial_{M}-i \tilde{g}_{5} \tilde{q}_{\tilde{\psi}} C_{M}(x, y)\right)-m_{\tilde{\psi}}\right\} \tilde{\psi}(x, y) \\
& \rightarrow \overline{\tilde{\psi}}(x, y+2 \pi R)\left\{i \Gamma^{M}\left(\partial_{M}-i \tilde{g}_{5} \tilde{q}_{\tilde{\psi}} C_{M}(x, y+2 \pi R)\right)-m_{\tilde{\psi}}\right\} \tilde{\psi}(x, y+2 \pi R) \\
& \quad=\overline{\tilde{\psi}}(x, y)\left\{i \Gamma^{M}\left(\partial_{M}-i \tilde{g}_{5} \tilde{q}_{\tilde{\psi}} C_{M}(x, y)\right)-m_{\tilde{\psi}}\right\} \tilde{\psi}(x, y)
\end{aligned}
$$

and

$$
\begin{gathered}
\overline{\tilde{\psi}}(x, y)\left\{i \Gamma^{M}\left(\partial_{M}-i \tilde{g}_{5} \tilde{q}_{\tilde{\psi}} C_{M}(x, y)\right)-m_{\tilde{\psi}}\right\} \tilde{\psi}(x, y) \\
\rightarrow \overline{\tilde{\psi}}(x,-y)\left\{i \Gamma^{M}\left(\partial_{M}-i \tilde{g}_{5} \tilde{q}_{\tilde{\psi}} C_{M}(x,-y)\right)-m_{\tilde{\psi}}\right\} \tilde{\psi}(x,-y) \\
=\overline{\tilde{\psi}}^{c}(x, y)\left\{i \Gamma^{\mu}\left(\partial_{\mu}+i \tilde{g}_{5} \tilde{q}_{\tilde{\psi}} C_{\mu}(x, y)\right)\right. \\
\left.\quad-i \Gamma^{5}\left(\partial_{5}+i \tilde{g}_{5} \tilde{q}_{\tilde{\psi}} C_{5}(x, y)\right)-m_{\tilde{\psi}}\right\} \tilde{\psi}^{c}(x, y) \\
=\overline{\tilde{\psi}}(x, y)\left\{i \Gamma^{M}\left(\partial_{M}-i \tilde{g}_{5} \tilde{q}_{\tilde{\psi}} C_{M}(x, y)\right)-m_{\tilde{\psi}}\right\} \tilde{\psi}(x, y),
\end{gathered}
$$

where we use the following relations concerning 4-component spinor fields $\tilde{\psi}$ :

$$
\begin{aligned}
& \overline{\tilde{\psi}}^{c} \gamma^{\mu} \partial_{\mu} \tilde{\psi}^{c}=\overline{\tilde{\psi}} \gamma^{\mu} \partial_{\mu} \tilde{\psi}, \quad \overline{\tilde{\psi}}^{c} \gamma^{5} \partial_{5} \tilde{\psi}^{c}=-\overline{\tilde{\psi}} \gamma^{5} \partial_{5} \tilde{\psi} \\
& \overline{\tilde{\psi}^{c}} \gamma^{\mu} \tilde{\psi}^{c}=-\overline{\tilde{\psi}} \gamma^{\mu} \tilde{\psi}, \quad \overline{\tilde{\psi}^{c}} \gamma^{5} \tilde{\psi}^{c}=\overline{\tilde{\psi}} \gamma^{5} \tilde{\psi}, \quad \overline{\tilde{\psi}} \tilde{\psi}^{c}=\overline{\tilde{\psi}} \tilde{\psi} .
\end{aligned}
$$

From (2.23), $\tilde{\psi}$ is given by the Fourier expansion:

$$
\tilde{\psi}(x, y)=\frac{1}{2 \sqrt{\pi R}} \sum_{n=-\infty}^{\infty}\left(\begin{array}{c}
\tilde{\xi}_{\alpha}^{(n)}(x) \\
i \dot{\tilde{\xi}}^{(n) \dot{\alpha}}(x)
\end{array}\right) e^{i \frac{2 \pi n+\beta}{2 \pi R} y},
$$

where $\tilde{\xi}_{\alpha}^{(n)}(x)$ are 4D 2-component spinor fields, and $\alpha$ and $\dot{\alpha}$ are spinor indices. Hereafter, we omit the spinor indices.

By inserting the mode expansions (2.27) and (2.31) into the 5D action $S_{5 \mathrm{D}}=$ $\int \mathscr{L}_{C} d^{5} x$, we obtain the following $4 \mathrm{D}$ action:

$$
\begin{aligned}
S_{4 \mathrm{D}}=\int d^{4} x[ & -\sum_{n=-\infty}^{\infty} \frac{1}{2} \tilde{\varphi}^{(n)}\left\{\square+\left(\frac{2 \pi n+\beta_{\tilde{\varphi}}-q_{\tilde{\varphi}} \theta}{2 \pi R}\right)^{2}+m_{\tilde{\varphi}}^{2}\right\} \tilde{\varphi}^{(n)} \\
& +\sum_{n=-\infty}^{\infty} \frac{1}{2}\left\{\tilde{\xi}^{(n)} i \sigma^{\mu} \partial_{\mu} \overline{\tilde{\xi}}^{(n)}+\overline{\tilde{\xi}}^{(n)} i \bar{\sigma}^{\mu} \partial_{\mu} \tilde{\xi}^{(n)}\right. \\
& +\left(\frac{2 \pi n+\beta_{\tilde{\psi}}-\tilde{q}_{\tilde{\psi}} \theta}{2 \pi R}+i m_{\tilde{\psi}}\right) \tilde{\xi}^{(n)} \tilde{\xi}^{(n)} \\
& \left.\left.+\left(\frac{2 \pi n+\beta_{\tilde{\psi}}-\tilde{q}_{\tilde{\psi}} \theta}{2 \pi R}-i m_{\tilde{\psi}}\right) \overline{\tilde{\xi}}^{(n)} \overline{\tilde{\xi}}^{(n)}\right\}\right]+\cdots,
\end{aligned}
$$

where $\sigma^{\mu}=(I, \boldsymbol{\sigma}), \bar{\sigma}^{\mu}=(I,-\boldsymbol{\sigma})\left(\boldsymbol{\sigma}=\left(\sigma_{x}, \sigma_{y}, \sigma_{z}\right)\right.$ are Pauli matrices $), \theta$ is the Wilson line phase defined by

$$
\theta=\tilde{g}_{5} \int_{-\pi R}^{\pi R} \frac{1}{\sqrt{2 \pi R}} C_{5}^{(0)} d y=\sqrt{2 \pi R} \tilde{g}_{5} C_{5}^{(0)},
$$


and the ellipsis stands for parts containing Kaluza-Klein modes of gauge bosons and the kinetic term of $C_{5}^{(0)}$. Note that the $U(1)$ gauge symmetry is broken by orbifolding, and $\theta$ is a remnant of the $U(1)$.

\section{Why hidden}

In order to obtain some hints to explore the origin of dark matter and the identity of inflaton and to address the reason for their existence, we search for an factor that it is hard to detect hidden particles based on the following assumptions.

- There is an extra gauge group $G_{\text {hidden }}$ other than the SM one $G_{\mathrm{SM}}$ (or some extension such as a grand unified group $G_{\mathrm{GUT}}$ ), and $G_{\text {hidden }}$ leaves little trace behind around the terascale.

- Hidden particles such as dark matter and inflaton possess gauge quantum numbers of $G_{\text {hidden }}$ or are some components of gauge bosons in a hidden sector, and they are gauge singlets of $G_{\mathrm{SM}}$ (or $\left.G_{\mathrm{GUT}}\right)$.

- The SM particles are gauge singlets of $G_{\text {hidden }}$.

Gauge quantum numbers are suitably assigned to construct a realistic model, but in most cases, it would be done without any foundation except for symmetry principle. We expect a reason or a mechanism that a subtle separation of gauge quantum numbers in the above assumptions is realized naturally, and propose a hypothesis that hidden particles can be separated according to gauge quantum numbers from the visible ones by the difference of BCs on extra dimensions 5

To embody our hypothesis, we consider a $5 \mathrm{D}$ theory with $G_{\mathrm{SM}} \times U(1)_{C}$ gauge group as an extension of the $\mathrm{SM}$ with an extra $U(1)$ gauge boson $C_{M}=C_{M}(x, y)$ and an extra matter $\tilde{\varphi}=\tilde{\varphi}(x, y)$. For simplicity, we pay attention to scalar fields and $U(1)$ gauge bosons and treat the Lagrangian density,

$$
\begin{aligned}
\mathscr{L}_{5 \mathrm{D}} & =\left(D_{M} H\right)^{*}\left(D^{M} H\right)-m_{H}^{2}|H|^{2}-\frac{1}{4} B_{M N} B^{M N} \\
& +\left(D_{M} \tilde{\varphi}\right)^{*}\left(D^{M} \tilde{\varphi}\right)-m_{\tilde{\varphi}}^{2}|\tilde{\varphi}|^{2}-\frac{1}{4} C_{M N} C^{M N} \\
& -\lambda\left(|H|^{2}\right)^{2}-\lambda_{\tilde{\varphi}}\left(|\tilde{\varphi}|^{2}\right)^{2}-\lambda_{\text {mix }}|H|^{2}|\tilde{\varphi}|^{2}+\cdots,
\end{aligned}
$$

where $H=H(x, y)$ is $5 \mathrm{D}$ complex scalar field containing the SM Higgs doublet as its zero mode $\left(H^{(0)}\right)$, and $\lambda, \lambda_{\tilde{\varphi}}$ and $\lambda_{\text {mix }}$ are quartic couplings of scalar fields.

If $B_{M}$ (the $5 \mathrm{D}$ extension of the $U(1)_{Y}$ gauge boson in the SM) satisfies the BCs such as (2.4) and (2.5) and $C_{M}$ satisfies the BCs such as (2.18) and (2.19), $H$ and $\tilde{\varphi}$

\footnotetext{
${ }^{5}$ According to a similar idea that a dark matter possesses different features from the SM particles on extra dimensions, a truncated-inert-doublet model has been constructed that the SM ones belong to $Z_{2}$ even zero modes and the dark matter is one of $Z_{2}$ odd zero modes on a warped extra dimension [3].
} 
cannot own both non-zero $U(1)$ charges. In other words, $H$ is separated from $\tilde{\varphi}$ in gauge interactions through the difference of BCs.

After the dimensional reduction, we obtain the following 4D Lagrangian density for zero modes $H^{(0)}, \tilde{\varphi}^{(0)}, B_{\mu}^{(0)}$ and $C_{5}^{(0)}$, at the tree level,

$$
\begin{aligned}
\mathscr{L}_{4 \mathrm{D}}^{(0)} & =\left(D_{\mu}^{(0)} H^{(0)}\right)^{*}\left(D^{(0) \mu} H^{(0)}\right)-m_{H}^{2}\left|H^{(0)}\right|^{2}-\frac{1}{4} B_{\mu \nu}^{(0)} B^{(0) \mu \nu} \\
& +\frac{1}{2} \partial_{\mu} \tilde{\varphi}^{(0)} \partial^{\mu} \tilde{\varphi}^{(0)}-\frac{1}{2}\left\{m_{\tilde{\varphi}}^{2}+\left(\frac{\beta_{\tilde{\varphi}}-\tilde{q}_{\tilde{\varphi}} \theta}{2 \pi R}\right)^{2}\right\}\left(\tilde{\varphi}^{(0)}\right)^{2}+\frac{1}{2} \partial_{\mu} C_{5}^{(0)} \partial^{\mu} C_{5}^{(0)} \\
& -\lambda\left(\left|H^{(0)}\right|^{2}\right)^{2}-\frac{1}{4} \lambda_{\tilde{\varphi}}\left(\tilde{\varphi}^{(0)}\right)^{4}-\frac{1}{2} \lambda_{\text {mix }}\left|H^{(0)}\right|^{2}\left(\tilde{\varphi}^{(0)}\right)^{2}+\cdots
\end{aligned}
$$

where we use the Fourier expansion (2.10) for $H$ and (2.27) for $\tilde{\varphi}$.

As seen from (3.2), $C_{5}^{(0)}$ is massless at the tree level. After receiving radiative corrections, the effective potential relating to $C_{5}^{(0)}$ is induced and $C_{5}^{(0)}$ acquires a mass through the Hosotani mechanism [4,5]. Concretely, the one-loop effective potential for the Wilson line phase $\theta\left(=\sqrt{2 \pi R} \tilde{g}_{5} C_{5}^{(0)}\right)$ is derived as

$$
\begin{aligned}
V_{\mathrm{eff}}[\theta] & =-\frac{1}{2} \int \frac{d^{4} p_{\mathrm{E}}}{(2 \pi)^{4}} \sum_{n=-\infty}^{\infty} \ln \left\{p_{\mathrm{E}}^{2}+m_{\tilde{\varphi}}^{2}+\left(\frac{2 \pi n+\beta_{\tilde{\varphi}}-\tilde{q}_{\tilde{\varphi}} \theta}{2 \pi R}\right)^{2}\right\} \\
& =E_{0}-\frac{3}{64 \pi^{6} R^{4}} \sum_{n=1}^{\infty}\left(\frac{1}{n^{5}}+\frac{r_{\tilde{\varphi}}}{n^{4}}+\frac{r_{\tilde{\varphi}}^{2}}{3 n^{3}}\right) e^{-n r_{\tilde{\varphi}}} \cos \left\{n\left(\beta_{\tilde{\varphi}}-\tilde{q}_{\tilde{\varphi}} \theta\right)\right\},
\end{aligned}
$$

where $p_{\mathrm{E}}$ is a $4 \mathrm{D}$ Euclidean momentum, $E_{0}$ is a $\theta$-independent constant and $r_{\tilde{\varphi}}=$ $2 \pi R m_{\tilde{\varphi}}$. The physical vacuum is realized at $\beta_{\tilde{\varphi}}-\tilde{q}_{\tilde{\varphi}} \theta=0$ and $C_{5}^{(0)}$ decouples in the low-energy theory, if $R$ is small enough, by acquiring the mass of $O(1 / R)$.

The scalar field $\tilde{\varphi}^{(0)}(x)$ survives in a post-SM at the terascale for $\beta_{\tilde{\varphi}}-\tilde{q}_{\tilde{\varphi}} \theta=$ 0 and $m_{\tilde{\varphi}}<O(1) \mathrm{TeV}$, and we find that our Lagrangian density agrees with that containing a dark matter in a specific model called the New Minimal Standard Model (NMSM) [6, 7]. Then, $\tilde{\varphi}^{(0)}(x)$ becomes a possible candidate of dark matter.

The $\tilde{\varphi}^{(0)}(x)$ couples to the SM Higgs doublet through the quartic interaction $-(1 / 2) \lambda_{\text {mix }}\left|H^{(0)}\right|^{2}\left(\tilde{\varphi}^{(0)}\right)^{2}$. In the presence of this term as the Higgs portal, the running of $\lambda$ based on the renormalization group equation changes compared with that in the SM, and the vacuum stability of Higgs potential can be improved [7, 8].

Here, as a complementary comment on our hypothesis, we state a feature that matters are not necessarily classified into the visible ones and the hidden ones, even if a system has two $U(1)$ gauge bosons $B_{M}$ and $C_{M}$ with different types of $B C$ s, because there can exist particles that possess both $U(1)$ charges. Let us show it using a model described by the Lagrangian density,

$$
\mathscr{L}_{\tilde{\varphi}_{a}}=\sum_{a=1,2}\left\{\left(D_{M} \tilde{\varphi}_{a}\right)^{*}\left(D^{M} \tilde{\varphi}_{a}\right)-m_{\tilde{\varphi}_{a}}^{2}\left|\tilde{\varphi}_{a}\right|^{2}\right\}-\frac{1}{4} B_{M N} B^{M N}-\frac{1}{4} C_{M N} C^{M N}
$$

where $D_{M}=\partial_{M}-i g_{5} q_{\tilde{\varphi}_{a}} B_{M}-i \tilde{g}_{5} \tilde{q}_{\tilde{\varphi}_{a}} C_{M}$ for a pair of complex scalar fields $\tilde{\varphi}_{a}=$ $\tilde{\varphi}_{a}(x, y)(a=1,2)$. In case that $q_{\tilde{\varphi}_{1}}=q_{\tilde{\varphi}_{2}}, \tilde{q}_{\tilde{\varphi}_{1}}=-\tilde{q}_{\tilde{\varphi}_{2}}$ and $m_{\tilde{\varphi}_{1}}=m_{\tilde{\varphi}_{2}}, \mathscr{L}_{\tilde{\varphi}_{a}}$ is a 
single-valued function under the BCs (2.4), (2.5), (2.18), (2.19) and

$$
\tilde{\varphi}_{a}(x, y+2 \pi R)=e^{i \tilde{\varphi}_{\tilde{\varphi}}} \tilde{\varphi}_{a}(x, y), \quad \tilde{\varphi}_{1}(x,-y)=\eta_{\tilde{\varphi}} \tilde{\varphi}_{2}(x, y),
$$

where $\beta_{\tilde{\varphi}}$ takes 0 or $\pi$ and $\eta_{\tilde{\varphi}}$ takes 1 or -1 . We refer to the $U(1)$ gauge symmetry concerning the BCs (2.18), (2.19) and (3.5) as an exotic $U(1)$ symmetry [9, 10] 6. Then, we find that $\tilde{\varphi}_{a}$ own both $U(1)$ gauge quantum numbers. A similar feature holds on a theory containing non-abelian gauge symmetries: matters can possess both gauge quantum numbers whose gauge bosons satisfy different types of BCs if the theory is vector-like.

\section{Gauge-higgs inflation}

We apply a model with conjugate BCs on a gauge-Higgs inflation scenario. Let us consider a gravity theory coupled to a $U(1)_{C}$ gauge theory defined on a $5 \mathrm{D}$ space-time whose classical background is $M^{4} \times S^{1} / Z_{2}$. The starting action is given by

$$
\begin{aligned}
S_{5 \mathrm{D}}^{\mathrm{gr}} & =\int d^{5} x \sqrt{-\hat{g}_{5}}\left[\frac{1}{16 \pi G_{5}} \hat{R}_{5}-\frac{1}{4} \hat{g}^{M P} \hat{g}^{N L} C_{M N} C_{P L}\right. \\
& \left.+\sum_{a=1}^{c_{1}} \overline{\tilde{\psi}}_{a}^{\mathrm{n}}\left(-i \hat{g}^{M N} \hat{\Gamma}_{M} \nabla_{N}-\mu_{a}\right) \tilde{\psi}_{a}^{\mathrm{n}}+\sum_{b=1}^{c_{2}} \overline{\tilde{\psi}}_{b}^{\mathrm{ch}}\left(-i \hat{g}^{M N} \hat{\Gamma}_{M} D_{N}-m_{b}\right) \tilde{\psi}_{b}^{\mathrm{ch}}\right],
\end{aligned}
$$

where $\hat{g}_{5}=\operatorname{det} \hat{g}_{M N}, \hat{g}^{M N}$ is the inverse of $5 \mathrm{D}$ metric $\hat{g}_{M N}, G_{5}$ is the $5 \mathrm{D}$ Newton constant, $\hat{R}_{5}$ is the 5D Ricci scalar, $C_{M N}=\partial_{M} C_{N}-\partial_{N} C_{M}, \hat{\Gamma}_{M}=E_{M}^{k} \Gamma_{k}\left(E_{M}^{k}=\right.$ $E_{M}^{k}(x, y)$ is the fünf bein, $\Gamma_{k}$ are $5 \mathrm{D}$ gamma matrices, and $k$ is the space-time index in the local Lorentz frame), $\nabla_{N}=\partial_{N}-(i / 4) \hat{\omega}_{N}^{k l} \Sigma_{k l}\left(\hat{\omega}_{N}^{k l}\right.$ is the spin connection and $\left.\Sigma_{k l}=i\left[\Gamma_{k}, \Gamma_{l}\right] / 2\right), D_{N}=\partial_{N}-(i / 4) \hat{\omega}_{N}^{k l} \Sigma_{k l}-i \tilde{g}_{5} \tilde{q}_{b} C_{N}$ for $\tilde{\psi}_{b}^{\text {ch }}, C_{N}$ is a $5 \mathrm{D} U(1)_{C}$ gauge boson in the hidden sector and we assume that it satisfies the conjugate BCs (2.18) and (2.19), $\tilde{\psi}_{a}^{\mathrm{n}}$ are neutral fermions, $\tilde{\psi}_{b}^{\mathrm{ch}}$ are $U(1)_{C}$ charged fermions whose $U(1)_{C}$ charge is $\tilde{q}_{b}$, and $c_{1}$ and $c_{2}$ stand for numbers of neutral and charged fermions, respectively. The $\tilde{g}_{5}$ is a $5 \mathrm{D}$ gauge coupling constant.

If the SM gauge bosons satisfy the ordinary BCs such as (2.4) and (2.5) and both $\tilde{\psi}_{a}^{\mathrm{n}}$ and $\tilde{\psi}_{b}^{\mathrm{ch}}$ satisfy the BCs (2.23) with $\beta_{a}$ and $\beta_{b}$ as a twisted phase $\left(\beta_{\tilde{\psi}}\right), \tilde{\psi}_{a}^{\mathrm{n}}$ and $\tilde{\psi}_{b}^{\mathrm{ch}}$ should be singlets of the SM gauge group, as a consequence in the previous section.

The BCs of $\hat{g}_{M N}$ are given by

$$
\begin{aligned}
& \hat{g}_{M N}(x, y+2 \pi R)=\hat{g}_{M N}(x, y), \\
& \hat{g}_{\mu \nu}(x,-y)=\hat{g}_{\mu \nu}(x, y), \quad \hat{g}_{\mu 5}(x,-y)=-\hat{g}_{\mu 5}(x, y), \quad \hat{g}_{55}(x,-y)=\hat{g}_{55}(x, y),
\end{aligned}
$$

and then the Fourier expansions of $\hat{g}_{M N}$ are presented as

$$
\hat{g}_{\mu \nu}(x, y)=\hat{g}_{\mu \nu}^{(0)}(x)+\sum_{n=1}^{\infty} \hat{g}_{\mu \nu}^{(n)}(x) \cos \frac{n y}{R},
$$

${ }^{6}$ The orbifolding due to these BCs is regarded as a variant of the diagonal embedding proposed in 11$]$. 


$$
\begin{aligned}
& \hat{g}_{\mu 5}(x, y)=\sum_{n=1}^{\infty} \hat{g}_{\mu 5}^{(n)}(x) \sin \frac{n y}{R} y, \\
& \hat{g}_{55}(x, y)=\hat{g}_{55}^{(0)}(x)+\sum_{n=1}^{\infty} \hat{g}_{55}^{(n)}(x) \cos \frac{n y}{R} .
\end{aligned}
$$

The spin connection $\hat{\omega}_{M}^{k l}$ satisfy the ordinary BCs such that

$$
\begin{aligned}
& \hat{\omega}_{M}^{k l}(x, y+2 \pi R)=\hat{\omega}_{M}^{k l}(x, y), \\
& \hat{\omega}_{\mu}^{k l}(x,-y)=\hat{\omega}_{\mu}^{k l}(x, y), \quad \hat{\omega}_{5}^{k l}(x,-y)=-\hat{\omega}_{5}^{k l}(x, y),
\end{aligned}
$$

and then the full Lagrangian density containing both visible and hidden sectors becomes a single-valued function on $S^{1} / Z_{2}$.

On the Minkowski background, $\hat{g}_{\mu \nu}^{(0)}$ takes the classical value such as $\left\langle g_{\mu \nu}^{(0)}\right\rangle=\eta_{\mu \nu}$, and other zero modes are assumed to have the following classical values:

$$
\left\langle\hat{g}_{55}^{(0)}\right\rangle=\phi^{2 / 3},\left\langle C_{5}^{(0)}\right\rangle=\frac{\theta}{\sqrt{2 \pi R} \tilde{g}_{5}},
$$

where $\phi$ is the radion and $\theta$ is the Wilson line phase. The Kaluza-Klein modes are assumed to have zero classical values.

According to a usual procedure, the following effective potential is obtained at the one-loop level,

$$
\begin{aligned}
& V(\rho, \theta)=\frac{3 L^{2} m^{6}}{2 \pi^{2} \rho^{2}}\left[-2 \zeta(5)+c_{1} \sum_{n=1}^{\infty}\left(\frac{1}{n^{5}}+r_{m} \frac{\rho^{1 / 3}}{n^{4}}+r_{m}^{2} \frac{\rho^{2 / 3}}{3 n^{3}}\right) e^{-n r_{m} \rho^{1 / 3}}\right. \\
& \left.+c_{2} \sum_{n=1}^{\infty}\left(\frac{1}{n^{5}}+\frac{\rho^{1 / 3}}{n^{4}}+\frac{\rho^{2 / 3}}{3 n^{3}}\right) e^{-n \rho^{1 / 3}} \cos \{n(\beta-\tilde{q} \theta)\}\right]+\frac{L^{2} m}{\rho^{1 / 3}} \tilde{a}+\cdots
\end{aligned}
$$

where we take common masses $\mu=\mu_{a}$ and $m=m_{b}$, a common twisted phase $\beta=\beta_{b}$ and a common charge $\tilde{q}=\tilde{q}_{b}$ for simplicity, $L=2 \pi R, \rho=L^{3} m^{3} \phi, \zeta(k)=\sum_{n=1}^{\infty} 1 / n^{k}$, $r_{m}=\mu / m$ and $\tilde{a}$ is some constant.

The above potential has a same form as that obtained in [12] except overall factor and $\beta$, and hence both radion and Wilson line phase are stabilized in case with $c_{1}>2+c_{2}$, and $\theta$ is, in particular, fixed as $\beta-\tilde{q} \theta=\pi$. Furthermore, the gauge-Higgs field $\theta$ can give rise to inflation in accord with the astrophysical data [13].

We need some modification of our model to explain the origin of the Big Bang after inflaton decays into the SM particles. The direct coupling between inflaton and some SM particles is necessary to produce radiations at a very early universe, but it is difficult due to the mismatch of BCs, as explained in the previous section. As a way out, if some SM particles or its extension form a pair of vector-like multiplet for $U(1)_{C}$ and satisfy the BCs such as (3.5) or counterparts of fermions, they can directly couple to $C_{5}^{(0)}$. For instance, if there exist two Higgs doublets $H_{a}$ as a vector-like pair of $U(1)_{C}$, there can appear the coupling such as $\tilde{g}_{5}^{2} \tilde{q}_{H}^{2}\left|H_{a}^{(0)}\right|^{2}\left(C_{5}^{(0)}\right)^{2}$. In this case, although the contributions from $H_{a}$ are added to the potential (4.10), $\theta$ might remain inflaton because they are not dominated. 


\section{Conclusions and discussions}

We have formulated 5D $U(1)$ gauge theories yielding conjugate $\mathrm{BCs}$ besides ordinary ones on $S^{1} / Z_{2}$. On the conjugate BCs, the $4 \mathrm{D}$ components of $U(1)_{C}$ gauge boson have odd $Z_{2}$ parities and their zero modes are projected out through the dimensional reduction. Then, the $U(1)_{C}$ gauge symmetry is broken down by orbifolding. In contrast, the fifth component of $U(1)_{C}$ gauge boson has even $Z_{2}$ parities, and its zero mode $C_{5}^{(0)}$ survives and becomes a dynamical field. It is massless at the tree level, but after receiving radiative corrections, the effective potential relating to $C_{5}^{(0)}$ is induced and $C_{5}^{(0)}$ acquires a mass of $O(1 / R)\left(R\right.$ is the radius of $\left.S^{1}\right)$ and decouple to the low-energy theory if $R$ is small enough. Matter fields transform into the charge conjugated ones under the $Z_{2}$ transformation, in case yielding the conjugate BCs. Then, only real fields such as real scalar fields and Majorana fermions appear after compactification.

We have shown that the separation of visible and hidden particles can be realized in gauge interactions using a $5 \mathrm{D}$ extension of the SM with an extra $U(1)$ gauge symmetry and an extra scalar field coexisting different types of BCs, and derived the Lagrangian density containing a dark matter in the NMSM. The zero mode of extra scalar field yielding the conjugate BCs becomes a possible candidate of dark matter.

Furthermore, we have applied a 5D gravity theory coupled to a $U(1)$ gauge theory with conjugate BCs on a gauge-Higgs inflation scenario and found that the effective potential containing the radion $\phi$ and Wilson line phase $\theta$ plays a role of an inflaton potential and $\theta$ becomes inflaton.

We have studied a model of dark matter and that of inflaton independently, to demonstrate the origin of such hidden particles clearly. We can combine them into a 5D gravity theory coupled to an extension of the SM (or grand unified theory) containing an extra $U(1)$ gauge boson $C_{M}$, an extra scalar field $\tilde{\varphi}$ and some extra fermions. In the combined theory, the zero mode of $\tilde{\varphi}$ becomes dark matter with $\beta_{\tilde{\varphi}}-\tilde{q}_{\tilde{\varphi}} \theta=0$, and that of $C_{5}$ becomes inflaton with $\beta-\tilde{q} \theta=\pi$. Then, $\theta$ must satisfy $\theta=\beta_{\tilde{\varphi}} / \tilde{q}_{\tilde{\varphi}}=(\beta-\pi) / \tilde{q}$, and hence the problem of origin of hidden particles is replaced by that of the selection for twisted phases $\left(\beta_{\tilde{\varphi}}, \beta\right)$ and $U(1)$ charges $\left(\tilde{q}_{\tilde{\varphi}}, \tilde{q}\right)$ to fix a suitable value of $\theta$. It would be interesting to construct a more realistic model based on the combined one. To generate the state of Big Bang after the end of inflation, it is necessary to couple some SM particles to inflaton and it can be realized, if some SM particles have an exotic $U(1)$ charge and they are related to ones with an opposite charge under the $Z_{2}$ reflection.

Finally, we give a comment on the right-handed neutrinos. Because the righthanded neutrinos are singlets of the SM gauge group and they have Majorana masses, we guess that they might be hidden matters obeying conjugate BCs. But it is difficult to realize it, because we cannot construct a $Z_{2}$ invariant term in 5D Lagrangian density to derive the 4D Yukawa interaction relating to neutrinos, due to the mismatch of BCs between the SM non-singlets and singlets. Nevertheless, it would also be interesting to examine the origin of the right-handed neutrinos from the viewpoint of BCs. 


\section{Acknowledgments}

Y. A. wishes to thank Iwanami Fu-Jukai for his Research grant and he would like to thank Prof. Katsushi Ito for his kind hospitality.

\section{References}

[1] A. Hebecker and J. March-Russell, Nucl. Phys. B 625, 128 (2002).

[2] N. Haba, Y. Kawamura and K. Oda, Phys. Rev. D 78, 085021 (2008).

[3] A. Ahmed, B. Grzadkowski, J. F. Gunion and Y. Jiang, JHEP 1510, 033 (2015).

[4] Y. Hosotani, Phys. Lett. B 126, 309 (1983).

[5] Y. Hosotani, Ann. of Phys. 190, 233 (1989).

[6] C. P. Burgess, M. Pospetov and T. ter Veldhuis, Nucl. Phys. B 619, 709 (2001).

[7] H. Davoudiasl, R. Kitano, T. Li and H. Murayama, Phys. Lett. B 609, 117 (2005).

[8] N. Haba, K Kanata and R. Takahashi, JHEP 1404, 029 (2014).

[9] Y. Kawamura and T. Miura, Int. J. Mod. Phys. A 26, 4405 (2011).

[10] Y. Kawamura and T. Miura, Int. J. Mod. Phys. A 27, 1250023 (2012).

[11] K. Kojima, K. Takenaga and T. Yamashita, Phys. Rev. D 84, 051701(R) (2011).

[12] Y. Abe, T. Inami, Y. Kawamura and Y. Koyama, Prog. Theor. Exp. Phys. 2014, $073 \mathrm{~B} 04$ (2014).

[13] Y. Abe, T. Inami, Y. Kawamura and Y. Koyama, Prog. Theor. Exp. Phys. 2015, 093B03 (2015). 